\title{
Treatment of at-level spinal cord injury pain with botulinum toxin A
}

\author{
Audrey Chun $\mathbb{1 0}^{1} \cdot$ Isaiah Levy ${ }^{2} \cdot{\text { Ajax Yang } \mathbb{1}^{3}}^{3}$ Andrew Delgado ${ }^{1,4} \cdot$ Chung-Ying Tsai $^{1} \cdot$ Eric Leung $^{5} \cdot$ Kristell Taylor $^{1}$. \\ Stephanie Kolakowsky-Hayner ${ }^{1} \cdot$ Vincent Huang $^{1} \cdot$ Miguel Escalon $^{1} \cdot$ Thomas N. Bryce $^{1}$
}

Received: 7 July 2019 / Revised: 23 August 2019 / Accepted: 1 September 2019

(c) The Author(s), under exclusive licence to International Spinal Cord Society 2019

\begin{abstract}
Study design Randomized, double-blinded, placebo-controlled, cross-over study.

Objective To explore whether botulinum toxin A (BoNTA) could be effective for treating at-level spinal cord injury (SCI) pain.

Setting Outpatient SCI clinic, New York, USA.

Methods Participants were randomized to receive subcutaneous injections of either placebo or BoNTA with follow-up (office visit, telephone, or e-mail) at 2, 4, 8, and 12 weeks to assess the magnitude of pain relief post injection. Crossover of participants was then performed. Those who received placebo received BoNTA, and vice versa, with follow-up at 2, 4, 8, and 12 weeks.

Results Eight participants completed at least one of the two crossover study arms. Four completed both arms. The median age of the eight participants was 45 years (range 32-61 years) and 75\% were male. All had traumatic, T1-L3 level, complete SCI. Although our data did not meet statistical significance, we noted a higher proportion of participants reporting a marked change in average pain intensity from baseline to 8 and 12 weeks post-BoNTA vs. post-placebo (33\% vs. $0 \%$ ). At 2 and 4 weeks post-BoNTA, almost all participants reported some degree of reduced pain, while the same was not seen postplacebo ( $83 \%$ vs. $0 \%$ ).
\end{abstract}

Conclusion The subcutaneous injection of BoNTA may be a feasible approach for the control of at-level SCI pain and is worthy of further study.

Sponsorship The onabotulinumtoxinA (BOTOX) used in this study was provided by Allergan (Irvine, CA).

\section{Introduction}

At-level spinal cord injury (SCI) pain is neuropathic pain perceived at the neurological level of injury (NLI) or within

Audrey Chun

audrey.s.chun@mssm.edu

1 Department of Rehabilitation and Human Performance, Icahn School of Medicine at Mount Sinai (ISMMS), New York, NY, USA

2 Icahn School of Medicine at Mount Sinai (ISMMS), New York, NY, USA

3 The Spine and Pain Institute of New York, New York, NY, USA

4 Graduate School of Biomedical Sciences, ISMMS, New York, NY, USA

5 Department of Physical Medicine and Rehabilitation, Department of Pain Medicine, Northwell Health Physician Partners, Bay Shore, NY, USA three levels below the NLI and is thought to be a result of damage to the spinal cord or its nerve roots [1]. It has been reported to occur in approximately one third of individuals with SCI [2, 3]. Various pharmacological agents used for treating at-level SCI pain have been shown to have only limited efficacy at best $[4,5]$. Therefore, a significant need for the investigation of potential new treatments for at-level SCI pain remains.

Botulinum toxin A (BoNTA) is a neurotoxin protein produced by the bacterium Clostridium botulinum. It acts at the neuromuscular junction and its onset of action occurs within 24-72 h after administration, with peak clinical effect occurring 4-6 weeks after administration, then 2-6 months of effect duration [6]. At the level of peripheral nerves, BoNTA may inhibit the synaptic release of local neuropeptides associated with pain transmission, such as glutamate, substance $\mathrm{P}$, and calcitonin gene-related peptide [7]. At the level of the central nervous system, retrograde effects of BoNTA on the spinal cord via axonal transport have been proposed $[8,9]$. 
BoNTA therapy has so far been shown to have some effectiveness in treating various peripherally mediated neuropathic pains, but the literature on BoNTA remains limited for treating centrally mediated pain [10]. To date, one randomized, double-blinded, placebo-controlled study has successfully been able to demonstrate that subcutaneous BoNTA may reduce neuropathic pain in persons with SCI. This study by Han et al. evaluated patients with neuropathic pain associated with SCI, both at-level $(n=9)$ and below-level $(n=29)$, and found participants demonstrated significantly reduced pain at 4 and 8 weeks after BoNTA injections compared with after placebo with saline. They also showed a marginal trend towards improvements on the physical health domain of the World Health Organization Quality of Life Instrument (WHOQOL-BREF abbreviated form) at 4 weeks postBoNTA vs. post-placebo [7].

BoNTA has been shown to have a favorable safety and tolerability profile across a broad spectrum of therapeutic uses [11, 12]. Adverse effects (AEs) associated with BoNTA are generally related to the mechanism of action of the toxin (e.g., dose-dependent focal weakness when injected intramuscularly). Other local side effects include temporary injection associated pain, edema, erythema, ecchymosis, headache, and short-term hyperesthesia. Systemic side effects include nausea, fatigue, malaise, rash, and flu-like symptoms [12]. In summary, a significant percentage of persons with SCI report at-level SCI pain but there is a lack of effective treatment to date, and BoNTA has shown preliminary evidence of effectiveness for neuropathic pain after SCI in one clinical trial while having a favorable safety and tolerability profile. Our study sought to further explore whether the subcutaneous injection of BoNTA could be a feasible approach for the control of at-level SCI pain.

\section{Methods}

\section{Study design and setting}

This study was designed as a randomized, double-blinded, placebo-controlled, crossover study. Institutional Review Board approval (IRB \#14-01107) was obtained prior to conducting any study related procedures and informed consent was obtained from each participant.

Participants received a subcutaneous injection of either normal saline (placebo) or BoNTA with follow-up (via office visit, telephone, or e-mail) at 2, 4, 8, and 12 weeks post injection (phase 1, P1). Crossover of participants was then performed. Those who received placebo received BoNTA, and vice versa, with follow-up at 2, 4, 8, and 12 weeks (phase 2, P2). Twelve weeks were determined as being the minimum required wash-out period based on the pharmacokinetics of BoNTA [6]. Participants were given the choice to start the P2 course at the time of 12-week follow-up for P1, or to defer the P2 course until a later time point.

All study activities took place in a large tertiary care hospital in the outpatient SCI rehabilitation clinic setting. Portions of this study have been accepted for poster presentation at the 2019 American Congress of Rehabilitation Medicine Annual Conference [13].

\section{Participants}

Participants were recruited via newsletter advertisements, physician recommendations, and Clinicaltrials.gov (NCT \#02736890) between 2016 and 2018. Participants were included if they reported chronic traumatic SCI (duration $>6$ months) and pain that has been: (1) present continuously for $\geq 1$ month, (2) of at least moderate average intensity over the prior week (numeric pain rating scale [NPRS] score $\geq 4 / 10$ ) and (3) diagnosed by an SCI provider as being at-level SCI pain with a high degree of certainty [14]. They were excluded if they were: $(1)<18$ years old, (2) pregnant, (3) with any contraindications to BoNTA (e.g., neuromuscular junction disease), (4) with a history of intolerance, hypersensitivity, or allergy to BoNTA, its preservatives, or the ethyl chloride used as an analgesic during injections, (5) with a history of BoNTA injections within the past 6 months, (6) with a history of coagulation disorder or current infection, (7) with insufficient command of English, and (8) unable to provide informed consent.

\section{Study procedure}

Consented participants underwent a pre-procedure evaluation, consisting of a focused history and physical exam, by a physician blinded to whether they would be BoNTA or placebo recipients. Each participant was asked to localize his or her area of worst pain, then to describe and rate the intensity of his or her pain before examination of the indicated painful area. Participants were then screened for the presence of neuropathic pain based on the Douleur Neuropathique 4 and SCI Pain Instrument questionnaires to supplement the focused history and exam [15]. The area of pain was marked using a skin marker and a plastic cut-out template for injection sites separated from each other by a $1 \mathrm{~cm}$ radius (Fig. 1).

To marked areas, participants received subcutaneous injections of either BoNTA or placebo. Each 100 unit vial of BoNTA was reconstituted with $4 \mathrm{ml}$ preservative-free sterile $0.9 \%$ normal saline solution, for a concentration of 5 units of BoNTA per $0.2 \mathrm{ml}$ injectate, as per manufacturer 


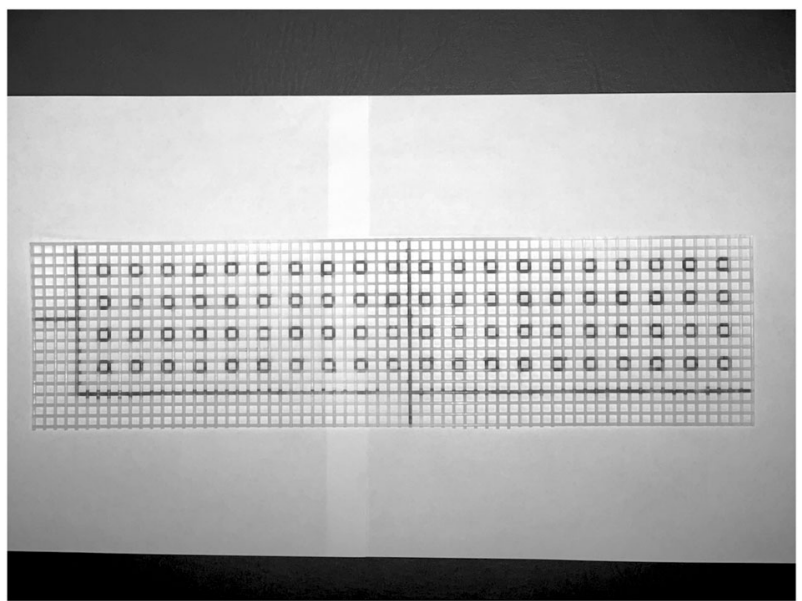

Fig. 1 Template used to administer botulinum toxin A (BoNTA) and placebo injections

recommendations. Placebo consisted of $0.2 \mathrm{ml}$ of the same preservative-free sterile $0.9 \%$ normal saline. Syringes were prefilled by an unblinded physician and research assistant in a separate location from where injections and assessments occurred. The physician administering the injection was blinded to syringe contents. The randomized treatment allocation for each patient was kept in a sealed envelope by another research assistant, separate from those preparing and administering injections. The area was sterilized with alcohol then localized topical anesthesia was provided with sterile ethyl chloride. Each injection was given using a 25gauge needle and administrators were allowed to provide up to 80 injections of 5 units each for a total maximum delivery of 400 units, per participant, per phase injection session. This maximum dose and injection number was determined based on the study team's review of the BoNTA manufacturer recommendations and the BoNTA literature to date at the time of protocol formation [16-18].

Following the injection procedure, participants were monitored for $\geq 5 \mathrm{~min}$ for immediate AEs. They were reeducated on potential AEs of BoNTA (e.g., local hyperesthesia, weakness, erythema), including all serious ones for which they should seek emergency care (e.g., respiratory distress, systemic illness), and provided with contact information for any questions or concerns that they may develop afterwards. Neither participants nor their insurance providers were charged for the BoNTA and participants did not receive any monetary reimbursement for participation. The BoNTA was stored in a locked refrigerator $\left(2-8{ }^{\circ} \mathrm{C}\right)$, labeled in a location separate from the outpatient SCI clinic.

\section{Outcomes}

Our primary outcome measure was patient reported average pain intensity over the prior week in their area of worst pain as indicated above. This measure is part of the International SCI Pain Basic Data Set (ISCIPBDS), whose validity and utility in individuals with SCI have been demonstrated, and it is among the measures recommended for use in SCI clinical trials [19-21]. Participants were asked to rate their average pain using the $0-10$ NPRS from 0 ("no pain") to 10 ("worst pain imaginable"). Secondary outcome measures included other ISCIPBDS items, such as the degree to which pain interfered with: (1) day to day activities, (2) mood, and (3) sleep, all on scales from 0 ("no interference") to 10 ("extreme interference"). We defined "marked improvement" as a $\geq 3$-point difference based on the minimal clinical important difference (MCID) for the NPRS being $\geq 3$ points [22]. At each follow-up time point, participants were asked to quantify the change in quality of life (QOL) appreciated after injections on a 7-point scale based on the Patient Global Impression of Change scale [19]. Lastly, participants were asked about and monitored for AEs as described above.

\section{Statistical analyses}

All collected data were stored in a password-protected REDCap database [23] and all hard copy forms were kept in locked files in the offices of research staff. Statistical analyses were performed using SPSS version 22.0. Power analyses were conducted based on the primary outcome of change in pain intensity from baseline. We determined that MCID on the NPRS would be $\geq 3$ points, that $30 \%$ of participants in the placebo group would have positive response to treatment, and that $40 \%$ more participants (i.e., $70 \%$ ) would respond in the treatment group [16, 22, 24]. Assuming a two-sided alpha of 0.05 , a sample size of 28 would provide $86 \%$ power. Assuming a $15 \%$ drop out rate, a sample size of 32 participants was deemed necessary for sufficient power to detect treatment effect. Our study failed to meet target sample size therefore it was determined that our findings would be best presented as a descriptive case series and descriptive statistics were used for participant characteristics and all outcomes.

\section{Results}

\section{Participant flow and characteristics}

Among 11 enrolled and consented participants, 3 did not proceed to injections because they were deemed ineligible for the study during the pre-procedure history and physical or they declined participation in the study at this point. Among the eight participants who proceeded, five started the study with BoNTA. All participants finished the P1 arm of the study. A single BoNTA recipient withdrew from the 


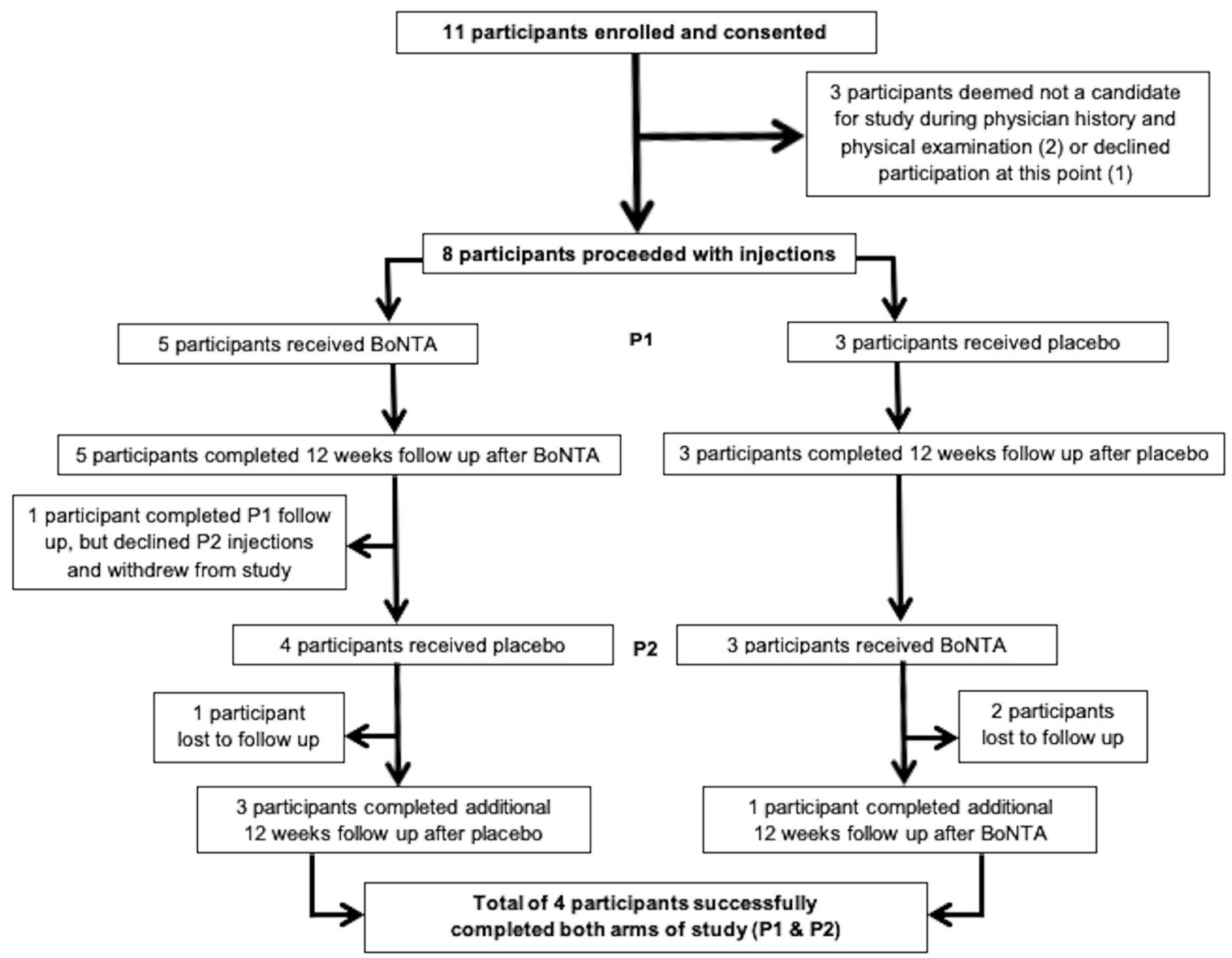

Fig. 2 Study participant flow and numbers lost to follow-up

study prior to starting the $\mathrm{P} 2$ arm, reporting increased pain after completing P1 (participant was blinded to whether BoNTA or placebo was given). One participant starting the study with BoNTA and two starting with placebo were lost to follow-up during P2. A total of four participants finished both P1 and P2 arms (Fig. 2). Participants had a median age of 45 years (range 32-61 years) and were predominantly male (75\%). All had traumatic, thoracic T1 to lumbar L3 level, complete SCI (American Spinal Injury Association Impairment Scale Grade A). Median duration of injury was 8 years (range 2-26 years). Table 1 shows detailed participant demographics and injury characteristics.

\section{Average pain intensity over the past week}

Participants A-E started with BoNTA injections at P1. Participants $\mathrm{F}-\mathrm{H}$ received BoNTA at time of crossover $\mathrm{P} 2$. For P1 and P2, a total six participants completed postBoNTA follow-up and six completed post-placebo followup. Participant A reported marked improvement in average pain intensity from baseline to 8 and 12 weeks postBoNTA, but not post-placebo. Participant B reported some degree of reduced pain at all weeks post-BoNTA.
Participant $\mathrm{C}$ reported some pain reduction at 2 weeks post-BoNTA, marked reduction at 4,8 , and 12 weeks, but not post-placebo. Participant D reported some pain reduction at 2 and 4 weeks post-BoNTA but at the 12week follow-up, withdrew from the study prior to receiving placebo, reporting increased pain after finishing P1. Participant E reported some pain reduction at 2, 4, and 8 weeks post-BoNTA, but received placebo for P2 some months after 12-week follow-up for P1 then was lost to follow-up. Participant $F$ reported marked pain reduction at 2 and 4 weeks post-BoNTA but not post-placebo. Participant $\mathrm{G}$ reported no pain reduction post-placebo, received BoNTA for P2 concurrently with 12-week follow-up for $\mathrm{P} 1$, then was lost to follow-up. Participant $\mathrm{H}$ reported no pain reduction post-placebo, received BoNTA for $\mathrm{P} 2$ some months after 12-week follow-up for P1, then was lost to follow-up (Table 2).

\section{Pain interference with day to day activities, mood, and sleep}

Participants A and C reported no marked improvements compared with baseline in day to day activities, mood or 
sleep, both post-BoNTA and post-placebo. Participant B reported a marked improvement in sleep at 2 weeks postBoNTA compared with baseline. Participant D reported markedly reduced pain interference with activities at 2 and 4 weeks post-BoNTA; markedly improved mood at 2 weeks post-BoNTA; and markedly improved sleep at 2 and 4 weeks post-BoNTA. Participant E reported markedly improved activities at 2 and 4 weeks post-BoNTA;

Table 1 Participant demographics and spinal cord injury (SCI) characteristics (total $n=8$ )

\begin{tabular}{lc}
\hline Demographics & \\
\hline Age at examination (years), median (min-max) & $45(32-61)$ \\
Sex, $N(\%)$ & $6(75 \%)$ \\
Male & $2(25 \%)$ \\
Female & \\
Race, $N$ (\%) & $2(25 \%)$ \\
Black or African American & $2(25 \%)$ \\
White or Caucasian & $3(37.5 \%)$ \\
Latino or Hispanic & $1(12.5 \%)$ \\
Unknown or not reported & $8(2-26)$ \\
\hline Injury characteristics & $8(100 \%)$ \\
\hline Duration of injury (years), median (min-max) & \\
Cause of injury, $N$ (\%) & $4(50 \%)$ \\
$\quad$ Traumatic & $4(50 \%)$ \\
Spinal cord injury (SCI) category, $N(\%)$ & \\
$\quad$ Thoracic T1-T6 paraplegia & \\
Thoracic T7-lumbar L3 paraplegia & \\
American Spinal Injury Association Impairment & \\
Scale (AIS) Grade, $N(\%)$ & \\
AIS A (complete injury) & \\
\hline
\end{tabular}

markedly improved mood at 2, 4, and 8 weeks postBoNTA; and markedly improved sleep at 4 weeks postBoNTA. Participant $F$ reported markedly improved activities at all weeks post-BoNTA; markedly improved mood at 2, 4, and 8 weeks post-BoNTA; and markedly improved sleep at all weeks post-BoNTA (Table 3).

\section{Change in quality of life following injections}

Participant A reported his QOL was "moderately better, with a slight but noticeable change" at 8 and 12 weeks post-BoNTA. Participant B reported his QOL was "somewhat better" at 2 and 4 weeks post-BoNTA. Participant C reported "somewhat" better QOL at 4 weeks and "moderately" better QOL at 12 weeks post-BoNTA (item assessment missed at 8 weeks). Participant D reported QOL that was "moderately better, with definite improvement" at 2 weeks post-BoNTA, but progressively reduced QOL at 4, 8, and 12 weeks. Participant E reported his QOL was "somewhat" better 2 weeks postBoNTA but reported minimal improvements at 4,8 , and 12 weeks. Participant F rated his QOL as "moderately better, with definite improvement" at 2 and 4 weeks postBoNTA, and "somewhat better" at 8 weeks post-BoNTA (Table 4).

\section{Adverse events}

One participant attributed an AE related to BoNTA injection itself: Participant D, who initially reported some improvements in pain, activities, mood, sleep, and QOL post-BoNTA, reported that overall his pain worsened at 12 weeks and withdrew from the study (Tables 2-4). Two participants reported an $\mathrm{AE}$ during the course of the study
Table 2 Participant reported average pain intensity over the past week (primary outcome)

\begin{tabular}{|c|c|c|c|c|c|c|c|c|}
\hline & \multicolumn{8}{|c|}{ Pain intensity in the past week? $(0-10)$} \\
\hline & \multicolumn{5}{|c|}{ Botulinum toxin A (BoNTA) then placebo } & \multicolumn{3}{|c|}{$\begin{array}{l}\text { Placebo } \\
\text { then BoNTA }\end{array}$} \\
\hline & A & $\mathrm{B}$ & $\mathrm{C}$ & $\mathrm{D}$ & $\mathrm{E}$ & $\mathrm{F}$ & G & $\mathrm{H}$ \\
\hline Baseline (phase 1-week 0) & 5 & 9 & 8 & 8 & 8 & 8 & 8 & 8 \\
\hline Initial 2-week follow-up & 5 & 7 & 7 & 6 & 7 & 8 & 8 & 8 \\
\hline Initial 4-week follow-up & 5 & 6 & 3 & 7 & 7 & 8 & 8 & 8 \\
\hline Initial 8-week follow-up & 2 & 8 & 4 & 8 & 6 & 8 & 8 & 8 \\
\hline Initial 12-week follow-up & 2 & 8 & 2 & 8 & 8 & 8 & 8 & 8 \\
\hline Baseline (phase 2-week 0) & & & & & 10 & & & 7 \\
\hline Crossover 2-week follow-up & 8 & 8 & 8 & \multirow{4}{*}{$\begin{array}{l}\text { Participant } \\
\text { withdrew }\end{array}$} & Lost to & 5 & \multirow{4}{*}{\multicolumn{2}{|c|}{$\begin{array}{l}\text { Lost t } \\
\text { fol- } \\
\text { low-u }\end{array}$}} \\
\hline Crossover 4-week follow-up & 8 & 8 & 8 & & follow-up & 5 & & \\
\hline Crossover 8-week follow-up & 8 & 5 & 7 & & & 6 & & \\
\hline Crossover 12 -week follow-up & 7 & 8 & 9 & & & 7 & & \\
\hline
\end{tabular}

Intensity reported using numeric pain rating scale, from 0 ("no pain") to 10 ("worst pain imaginable") 
Table 3 Participant reported pain interference with day to day activities, mood, and sleep

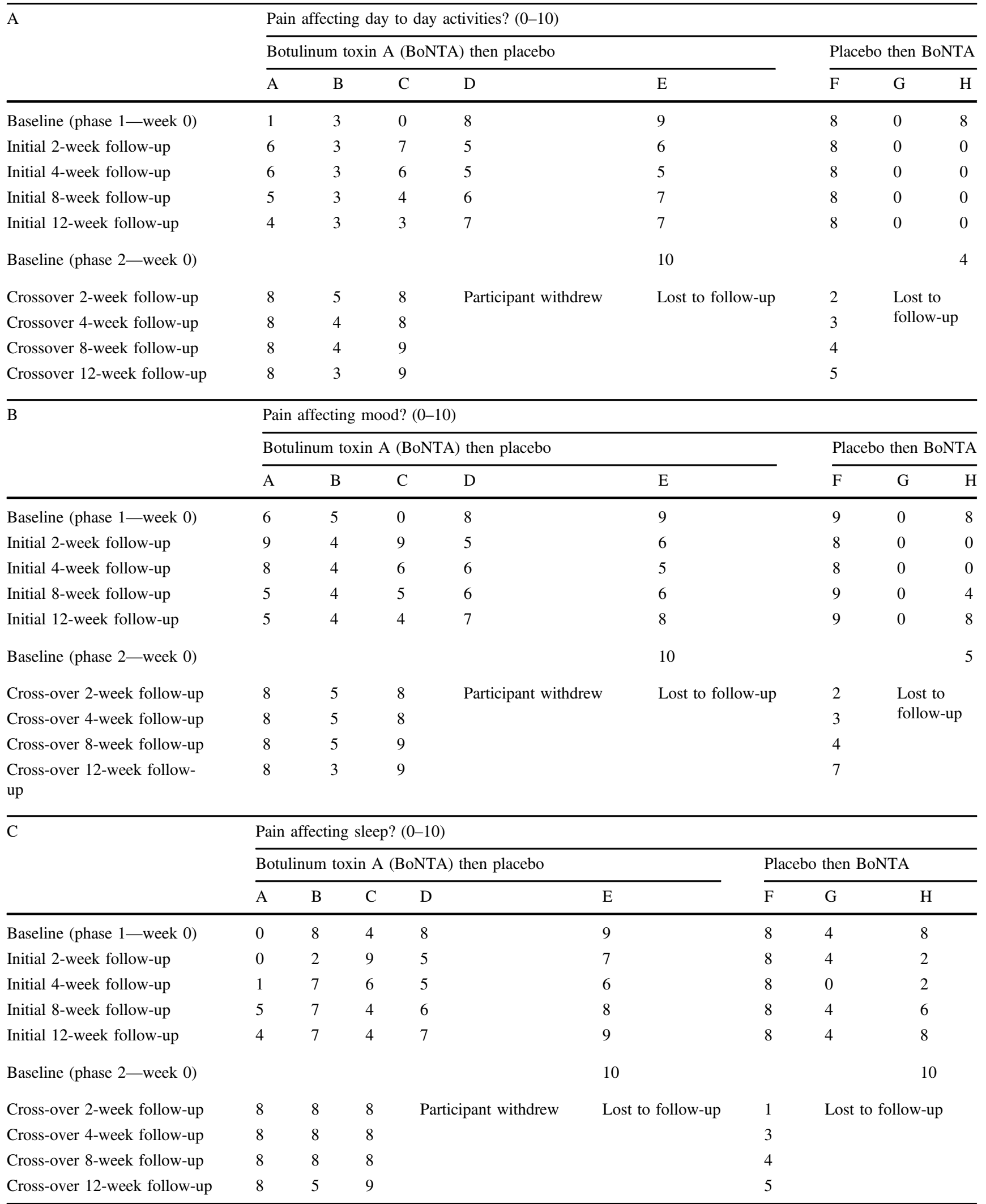

Degree of interference reported using a numeric scale with endpoints of 0 ("no interference") to 10 ("extreme interference") 
Table 4 Participant reported change in quality of life following injections

\begin{tabular}{|c|c|c|c|c|c|c|c|c|}
\hline & \multicolumn{8}{|c|}{ Change in quality of life? (0-6) } \\
\hline & \multicolumn{5}{|c|}{ Botulinum toxin A (BoNTA) then placebo } & \multicolumn{3}{|c|}{ Placebo then BoNTA } \\
\hline & A & $\mathrm{B}$ & $\mathrm{C}$ & $\mathrm{D}$ & $\mathrm{E}$ & $\mathrm{F}$ & G & $\mathrm{H}$ \\
\hline Baseline (phase 1-week 0) & $\mathrm{N} / t$ & & & & & & & \\
\hline Initial 2-week follow-up & 0 & 3 & 0 & 5 & 3 & 0 & 1 & 0 \\
\hline Initial 4-week follow-up & 0 & 3 & 3 & 4 & 2 & 0 & 1 & 0 \\
\hline Initial 8-week follow-up & 4 & 0 & a & 2 & 2 & 0 & 0 & 0 \\
\hline Initial 12-week follow-up & 4 & 0 & 4 & 1 & 1 & 0 & 0 & 0 \\
\hline Baseline (phase 2-week 0) & $\mathrm{N} /$ & & & & & & & \\
\hline Crossover 2-week follow-up & 0 & 0 & 0 & Participant withdrew & Lost to follow-up & 5 & Lo & $v$-up \\
\hline Crossover 4-week follow-up & 0 & 0 & 0 & & & 5 & & \\
\hline Crossover 8-week follow-up & 0 & 0 & 0 & & & 3 & & \\
\hline Crossover 12-week follow-up & 0 & 0 & 0 & & & 1 & & \\
\hline
\end{tabular}

Amount of change in quality of life appreciated after injections on a scale of 0-6, with corresponding verbal descriptions as below:

$0=$ "no change, or condition got worse"

$1=$ "almost the same, hardly any change at all"

2 = "a little better, but no noticeable change"

$3=$ "somewhat better, but the change has not made any real difference"

$4=$ "moderately better, a slight but noticeable change"

$5=$ "moderately better, a definite improvement that has made a real worthwhile difference"

$6=$ "a great deal better, a considerable improvement that has made all the difference"

atem data could not be obtained for participant $\mathrm{C}$ at 8 weeks follow-up for phase 1 (P1)

(Participant A reported "thrombosis of a preexisting inferior vena cava filter" and Participant $\mathrm{H}$ reported "a fever from a cold") but both denied feeling the events were related to the study.

\section{Discussion}

Our study sought to explore whether the subcutaneous injection of BoNTA is a feasible approach for the control of at-level SCI pain. This study was designed as a randomized, double-blinded, placebo-controlled, crossover study. However, we failed to meet target sample size and determined our findings would be best presented as a descriptive case series. Our data did not meet statistical significance and we were not statistically powered to, but we did note various patterns of improved pain, activities, mood, and sleep among participants after BoNTA.

More participants reported a marked change in average pain intensity from baseline to 8 and 12 weeks post-BoNTA vs. post-placebo (33\% vs. 0\%). At 2 and 4 weeks postBoNTA, almost all participants reported some degree of reduced pain; the same was not seen post-placebo (83\% vs. 0\%) (Table 2). This is consistent with Han et al.'s findings that, at 4 and 8 weeks after BoNTA injections, visual analog scale scores for pain were significantly reduced by $18.6 \pm$ 16.8 and $21.3 \pm 26.8$, but only reduced by $2.6 \pm 14.6$ and $0.3 \pm 19.5$ after placebo injections [7].

More participants reported markedly reduced pain interference with day to day activities at 2 and 4 weeks postBoNTA vs. post-placebo (50\% vs. $17 \%$ ). More participants reported markedly reduced pain interference with mood post-BoNTA vs. post-placebo at $2(50 \%$ vs. 17\%), 4 (33\% vs. $17 \%)$, and 8 (33\% vs. 17\%) weeks. More participants reported markedly reduced pain interference with sleep post-BoNTA vs. post-placebo at 2 (50\% vs. $17 \%), 4(50 \%$ vs. $33 \%), 8(17 \%$ vs. $0 \%)$ and $12(17 \%$ vs. $0 \%)$ weeks (Table 3). Lastly, more participants reported at least moderate improvements in QOL post-BoNTA vs. post-placebo at $2(33 \%$ vs. $0 \%)$ and 4 (33\% vs. $0 \%), 8(17 \%$ vs. $0 \%)$, and 12 (33\% vs. $0 \%$ ) weeks (Table 4$)$. This too supports Han et al.'s trend towards improvements at 4 weeks postBoNTA $(p=0.0521)$ on the physical health domain of the WHOQOL-BREF, which includes facets like activities of daily living and sleep [7].

Our data was statistically insufficient to draw conclusions regarding the effects of BoNTA on at-level SCI pain. Despite this, we managed to demonstrate several interesting patterns within our participants' reports on how they felt post-BoNTA. Participants also denied any significant AEs 
from receiving BoNTA injections, consistent with the drug's favorable safety and tolerability profile demonstrated in the literature to date $[11,12]$. One withdrew from the study reporting worsened pain following BoNTA injections, but it was later noted this participant had initially reported improvements in pain, activities, mood, sleep, and QOL post-BoNTA.

The largest limitation to our study was our failure to meet target sample size due to low recruitment and retention rates. BoNTA injections were offered for free but the time, planning, and costs required of participants to coordinate injection visits were inadequately accounted for. Coordinating follow-ups also proved to be difficult once participants received injections, possibly because patients lost incentives to continue participating. These issues could be better addressed with more resources for both improved recruitment and retention in the future. Based on our findings, we encourage the field to continue considering BoNTA as a potential option for treating at-level SCI pain. We further hope that sharing our limitations may facilitate additional investigations into this treatment method throughout the field.

\section{Conclusion}

Several encouraging patterns among participants with respect to self-reported average pain levels; pain interference with day to day activities, mood, and sleep; as well as overall QOL as related to pain were observed postBoNTA injections. These findings are consistent with the literature to date and incite us to continue investigating subcutaneous BoNTA as a feasible approach for the control of at-level SCI pain.

\section{Data availability}

Survey data are available from the corresponding author upon reasonable request.

Acknowledgements We thank Dr. Tiffany Wong (Department of Rehabilitation and Human Performance, ISMMS, New York, NY) for her assistance with injections during her SCI Fellowship.

Funding The onabotulinumtoxinA (BOTOX) used for this study was provided by Allergan (Irvine, CA). Funding source personnel had no involvement in the study design; collection, analysis and interpretation of the data; or the writing and submission of this paper for publication.

Author contributions All listed authors have met all of the following authorship criteria: (1) Conceived and/or designed the work that led to the submission, acquired data, and/or played an important role in interpreting the results. (2) Drafted or revised the paper and approved the final version. (3) Agreed to be accountable for all aspects of the work in ensuring that questions related to the accuracy or integrity of any part of the work are appropriately investigated and resolved.

\section{Compliance with ethical standards}

Conflict of interest The authors declare no conflict of interest.

Ethical approval We certify that all applicable institutional and governmental regulations concerning the ethical use of human volunteers were followed during the course of this research.

Publisher's note Springer Nature remains neutral with regard to jurisdictional claims in published maps and institutional affiliations.

\section{References}

1. Bryce TN, Biering-Sorensen F, Finnerup NB, Cardenas DD, Defrin R, Ivan E, et al. International Spinal Cord Injury Pain (ISCIP) Classification: Part 2. Initial validation using vignettes. Spinal Cord 2012;50:404-12.

2. Finnerup NB, Jensen MP, Norrbrink C, Trok K, Johannesen IL, Jensen TS, et al. A prospective study of pain and psychological functioning following traumatic spinal cord injury. Spinal Cord. 2016;54:816-21.

3. Siddall PJ, McClelland JM, Rutkowski SB, Cousins MJ. A longitudinal study of the prevalence and characteristics of pain in the first 5 years following spinal cord injury. Pain. 2003;103:249-57.

4. Finnerup NB, Attal N, Haroutounian S, McNicol E, Baron R, Dworkin $\mathrm{RH}$, et al. Pharmacotherapy for neuropathic pain in adults: a systematic review and meta-analysis. Lancet Neurol. 2015;14:162-73.

5. Guy SD, Mehta S, Casalino A, Cote I, Kras-Dupuis A, Moulin DE, et al. The CanPain SCI clinical practice guidelines for rehabilitation management of neuropathic pain after spinal cord: recommendations for treatment. Spinal Cord. 2016;54(Suppl 1):S14-23.

6. Elovic EB, E. Escaldi, S.V. Reyna, M. Chemodenervation With Botulinum Toxin. (2015) In Cuccurullo SJ (ed.), Physical medicine and rehabilitation board review. 3rd ed. (pp 869-71). New York, USA: Demos Medical.

7. Han ZA, Song DH, Oh HM, Chung ME. Botulinum toxin type A for neuropathic pain in patients with spinal cord injury. Ann Neurol. 2016;79:569-78.

8. Bach-Rojecky L, Lackovic Z. Central origin of the antinociceptive action of botulinum toxin type A. Pharm Biochem Behav. 2009;94:234-8.

9. Matak I, Lackovic Z. Botulinum toxin A, brain and pain. Prog Neurobiol. 2014;119-20:39-59.

10. Safarpour Y, Jabbari B. Botulinum toxin treatment of pain syndromes - an evidence based review. Toxicon. 2018;147:120-8.

11. Lu DW, Lippitz J. Complications of botulinum neurotoxin. Dis Mon. 2009;55:198-211.

12. Naumann M, Jankovic J. Safety of botulinum toxin type A: a systematic review and meta-analysis. Curr Med Res Opin. 2004;20:981-90.

13. Chun A, Levy I, Yang A, Delgado A, Tsai CY, Leung E, et al. Treatment of at-level spinal cord injury pain with botulinum toxin A. [Presentation] American Congress of Rehabilitation Medicine 96th Annual Conference. Chicago, IL, 7th November 2019.

14. Forchheimer MB, Richards JS, Chiodo AE, Bryce TN, DysonHudson TA. Cut point determination in the measurement of pain and its relationship to psychosocial and functional measures after 
traumatic spinal cord injury: a retrospective model spinal cord injury system analysis. Arch Phys Med Rehabil. 2011;92:419-24.

15. Bryce TN, Richards JS, Bombardier CH, Dijkers MP, Fann JR, Brooks L, et al. Screening for neuropathic pain after spinal cord injury with the spinal cord injury pain instrument (SCIPI): a preliminary validation study. Spinal Cord. 2014;52:407-12.

16. Yuan RY, Sheu JJ, Yu JM, Chen WT, Tseng IJ, Chang HH, et al. Botulinum toxin for diabetic neuropathic pain: a randomized double-blind crossover trial. Neurology. 2009;72:1473-8.

17. Xiao L, Mackey S, Hui H, Xong D, Zhang Q, Zhang D. Subcutaneous injection of botulinum toxin a is beneficial in postherpetic neuralgia. Pain Med. 2010;11:1827-33.

18. Wu CJ, Lian YJ, Zheng YK, Zhang HF, Chen Y, Xie NC, et al. Botulinum toxin type A for the treatment of trigeminal neuralgia: Results from a randomized, double-blind, placebo-controlled trial. Cephalalgia. 2012;32:443-50.

19. Bryce TN, Budh CN, Cardenas DD, Dijkers M, Felix ER, Finnerup NB, et al. Pain after spinal cord injury: an evidence-based review for clinical practice and research. Report of the National
Institute on Disability and Rehabilitation Research Spinal Cord Injury Measures meeting. J Spinal Cord Med. 2007;30:421-40.

20. Jensen MP, Widerstrom-Noga E, Richards JS, Finnerup NB, Biering-Sorensen F, Cardenas DD. Reliability and validity of the International Spinal Cord Injury Basic Pain Data Set items as selfreport measures. Spinal Cord. 2010;48:230-8.

21. Widerstrom-Noga E, Biering-Sorensen F, Bryce T, Cardenas DD, Finnerup NB, Jensen MP. et al. The international spinal cord injury pain basic data set. Spinal Cord. 2008;46:818-23.

22. Hanley MA, Jensen MP, Ehde DM, Robinson LR, Cardenas DD, Turner JA. et al. Clinically significant change in pain intensity ratings in persons with spinal cord injury or amputation. Clin $\mathrm{J}$ Pain. 2006;22:25-31.

23. Harris PA, Taylor R, Thielke R, Payne J, Gonzalez N, Conde JG. Research electronic data capture (REDCap)-a metadata-driven methodology and workflow process for providing translational research informatics support. J Biomed Inform 2009;42:377-81.

24. Beecher HK. The powerful placebo. J Am Med Assoc. 1955;159:1602-6. 